\title{
The mass profile of early-type galaxies in overdense environments: the case of the double source-plane gravitational lens SL2SJ02176-0513 ${ }^{\star}$
}

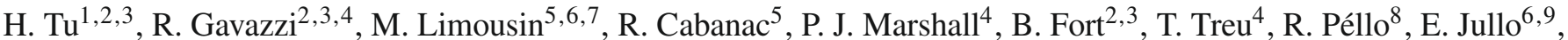 \\ J.-P. Kneib ${ }^{6,10}$, and J.-F. Sygnet ${ }^{2,3}$
}

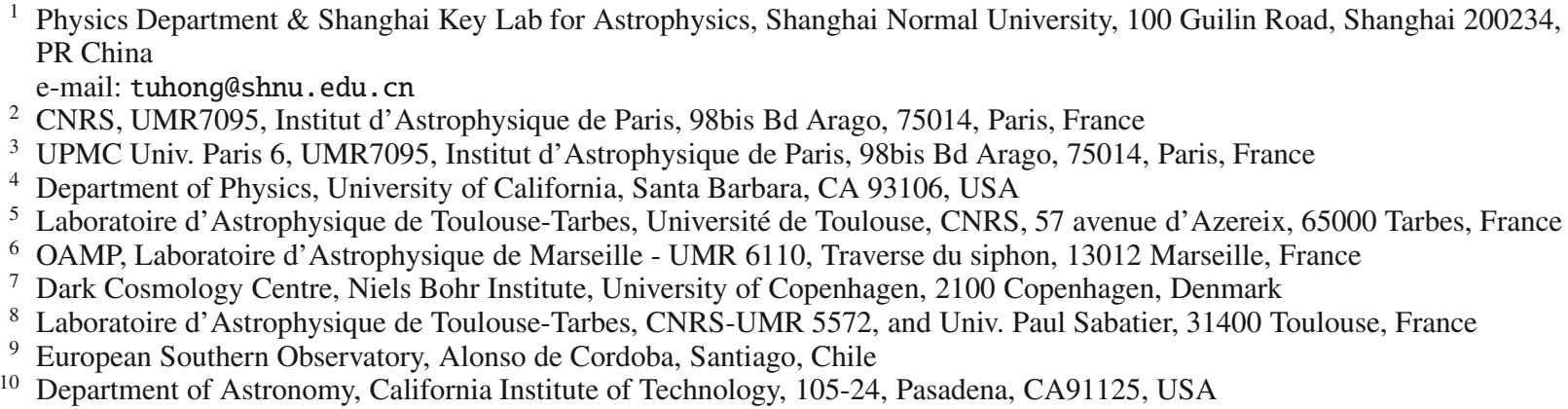

Received 27 February 2009 / Accepted 13 April 2009

\section{ABSTRACT}

\begin{abstract}
Context. The Strong Lensing Legacy Survey (SL2S) provides a sample of the strong lensing events associated with massive distant galaxies, some of which lie in the outskirts of galaxy groups and clusters.

Aims. We investigate the internal structure of early-type galaxies in overdense environments, where tidal forces are expected to alter dark matter haloes of infalling galaxies.

Methods. SL2SJ02176-0513 is a remarkable lens for the presence of two multiply-imaged systems at different redshifts lensed by a foreground massive galaxy at $z_{\text {lens }}=0.656$ : a bright cusp arc at $z_{\text {arc }}=1.847$ and an additional double-image system at an estimated redshift of $z_{\mathrm{dbl}} \sim 2.9$ based on photometry and lensing geometry. The system is located about $400 \mathrm{kpc}$ away from the centre of a massive group of galaxies. Mass estimates for the group are available from X-ray observations and satellite kinematics. Multicolour photometry provides an estimate of the stellar mass of the main lens galaxy. The lensing galaxy is modelled with two components (stars and dark matter), and we include the perturbing effect of the group environment and all available constraints.

Results. We find that classic lensing degeneracies, e.g. between external convergence and mass density slope, are significantly reduced with respect to standard systems and infer tight constraints on the mass density profile: (i) the dark matter content of the main lens galaxy is in line with that of typical galaxies $f_{\mathrm{dm}}\left(<R_{\mathrm{e}}\right)=0.41_{-0.06}^{+0.09}$; (ii) the required mass associated with the dark matter halo of the nearby group is consistent with X-ray $\left(\sigma_{\text {grp }}=550_{-240}^{+130}\right)$; (iii) accounting for the group contribution in the form of an external convergence, the slope of the mass density profile of the main lens galaxy alone is found to be $\alpha=-1.03_{-0.16}^{+0.22}$, consistent with the isothermal $(\alpha=-1)$ slope.

Conclusions. We demonstrate that multiple source plane systems together with good ancillary dataset can be used to disentangle local and environmental effects.
\end{abstract}

Key words. gravitational lensing - Galaxy: general - dark matter

\section{Introduction}

In the past thirty years, gravitational lensing has become a major astrophysical tool because of its unique ability to probe the densest regions of the universe independent of their emitted

\footnotetext{
* Based on observations obtained with MegaPrime/MegaCam, a joint project of CFHT and CEA/DAPNIA, at the Canada-France-Hawaii Telescope (CFHT) which is operated by the National Research Council (NRC) of Canada, the Institut National des Science de l'Univers of the Centre National de la Recherche Scientifique (CNRS) of France, and the University of Hawaii. This work is based in part on data products produced at TERAPIX and the Canadian Astronomy Data Centre as part of the Canada-France-Hawaii Telescope Legacy Survey, a collaborative project of $\mathrm{NRC}$ and CNRS.
}

radiation. Large imaging or spectroscopic surveys are becoming key players, in that context, by bringing numerous lensing events of all scales and spanning an increasingly broader range of masses and redshifts (e.g. Kochanek et al. 1999; Myers et al. 2003; Inada et al. 2008; Bolton et al. 2006, 2008a; Faure et al. 2008a; Marshall et al. 2008).

Among the imaging searches for lenses, the CFHT Legacy Survey ${ }^{1}$ stands out by combining depth in five photometric bands, excellent image quality $\left(\sim 0 ., 7-0 .{ }^{\prime} 8\right.$ median seeing) and an area of $170 \mathrm{deg}^{2}$ when completed. The Strong Lensing Legacy Survey (SL2S) ${ }^{2}$ aims at automatically detecting

\footnotetext{
1 http://www.cfht.hawaii.edu/Science/CFHLS/

${ }^{2}$ http://www-sl2s.iap.fr/
} 
gravitational lensing events in these data (Cabanac et al. 2007; Limousin et al. 2008), be they large arcs in groups and clusters or smaller, galaxy-scale lensing events (sometimes also referred to as "Einstein rings" to remind us that the lensed background source is extended, and therefore does, at some surface brightness level, produce a complete ring around the deflector). A detailed description of the ringfinder detection method will be given in a forthcoming paper (Gavazzi et al. 2009, in prep.).

One of the scientific questions that can be addressed in a direct and powerful manner by gravitational lensing is that of the dark matter halo profiles of early-type galaxies, the most common type of strong lenses. For example, gravitational lensing has been used to demonstrate the presence of dark matter haloes (e.g. Kochanek 1994; Treu \& Koopmans 2004), and to test the validity of the universal dark matter haloes predicted by numerical simulations (Navarro et al. 1997a; Gavazzi et al. 2007). When galaxies fall into an overdense environment, i.e. a cluster or a group, their dark matter haloes are expected to be stripped by tidal interactions (e.g. Dobke et al. 2007). Tidal stripping typically does not reach deep enough into the infalling galaxy to affect its luminous structure (e.g. Treu et al. 2003) until the galaxy reaches the densest regions, and therefore the effects are hardly detectable with classical astronomical probes. However, stripping of the outer halo begins much earlier during the infall and can be detected by gravitational lensing, for example by weak lensing analysis of cluster galaxies (Natarajan \& Kneib 1997; Geiger \& Schneider 1998; Natarajan et al. 2002a, 2007). Analyses of selected galaxy samples coupling lensing and dynamics have also suggested that haloes of satellite galaxies may be truncated and effectively steeper than those of isolated galaxies (e.g. Treu \& Koopmans 2002; Auger 2008; Treu et al. 2008). In turn, galaxy-scale strong lenses in the field of clusters, can be used to constrain the cluster mass distribution near the high magnification regions of the cluster (Tu et al. 2008; Limousin et al. 2008) and that of nearby structures (e.g. Kochanek \& Blandford 1991; King 2007; Tu et al. 2008).

Beyond the general problem of tidal truncation of dark matter haloes, the environment of lens galaxies has been a topic of intense debate in the lensing literature for a variety of reasons. One important question is the role of the environment in the measurement of Hubble's constant from lens time delays (e.g. Kochanek 2002; Kochanek \& Schechter 2004). In addition, the environment of lens galaxies must be understood if one wants to generalise the findings to the overall population of similar galaxies. It is known that multiply-imaged QSOs are often found behind overdense regions which have noticeable effects on the lensing configurations (e.g. Keeton et al. 1997; Fassnacht \& Lubin 2002; Auger et al. 2007; Momcheva et al. 2006; Oguri 2006; Williams et al. 2008). This preference for dense environments is also seen in the low-redshift SLACS sample (Treu et al. 2008). However, these authors showed that this preference is no more pronounced than for any massive elliptical galaxy, whether or not it is actually a lens. Within the errors, the preference for high-density environments is just a consequence of the clustering of massive galaxies. A somewhat similar trend is observed in the COSMOS survey (Faure et al. 2008b) although the use of weak lensing convergence map as a proxy for the environment makes the interpretation a bit more difficult. This preference for clustering is nicely illustrated by the results of Fassnacht et al. (2006) and Newton et al. (2008) which can be summarised as: $a$ good place to look for new lenses is the vicinity of known lenses.

In this paper, we present the analysis of an extraordinary system SL2SJ02176-0513 discovered by the CFHT Legacy Survey, and use it as a case study to investigate the effect of
Table 1. Summary of the most relevant observational quantities for SL2SJ02176-0513.

\begin{tabular}{|c|c|c|}
\hline Lens galaxy centre $(\mathrm{J} 2000)$ & $\begin{array}{l}\text { RA } \\
\text { Dec }\end{array}$ & $\begin{array}{l}02: 17: 37.136 \\
-05: 13: 29.41\end{array}$ \\
\hline Lens galaxy redshift & $z_{\text {lens }}$ & $0.6459 \pm 0.0003$ \\
\hline \multirow[t]{5}{*}{ Lens mags $\left(R<1^{\prime \prime}\right)$} & $u^{*}$ & $23.90 \pm 0.20$ \\
\hline & $g^{\prime}$ & $22.89 \pm 0.07$ \\
\hline & $r^{\prime}$ & $21.74 \pm 0.05$ \\
\hline & $i^{\prime}$ & $20.44 \pm 0.04$ \\
\hline & $z^{\prime}$ & $20.12 \pm 0.03$ \\
\hline \multirow[t]{2}{*}{ HST (Sérsic model mags) } & F606W & $20.90 \pm 0.05$ \\
\hline & $F 814 W$ & $19.71 \pm 0.01$ \\
\hline Effective radius $(F 814 W)$ & $R_{\mathrm{e}}$ & $\begin{array}{c}0.90 \pm 0.10 \\
6.20 \pm 0.7 h_{70}^{-1} \mathrm{kpc}\end{array}$ \\
\hline Sérsic model index $(F 814 W)$ & $n$ & 5.20 \\
\hline Rest frame $V$ band luminosity & $L_{V}$ & $1.36_{-0.08}^{+0.08} \times 10^{11} h_{70}^{-2} L_{\odot}$ \\
\hline light position angle $(F 814 W)$ & $\mathrm{PA}_{*}$ & $78 \pm 6 \mathrm{deg}$ \\
\hline light ellipticity $(F 814 W)$ & $\epsilon_{*}$ & $0.14 \pm 0.06$ \\
\hline "Photometric" stellar mass & $M_{*}$ & $2.1-4.1 \times 10^{11} M_{\odot}$ \\
\hline \multicolumn{3}{|l|}{ Tangential arc } \\
\hline$\ldots$ redshift & & $1.8470 \pm 0.0003$ \\
\hline$\ldots$ & $F 606 W_{\text {arc }}$ & $22.04 \pm 0.16$ \\
\hline & $F 814 W_{\text {arc }}$ & $22.19 \pm 0.13$ \\
\hline \multicolumn{3}{|l|}{ Double system (R1) } \\
\hline ... photom. redshift & $z_{\mathrm{dbl}}$ & $2.90_{-0.24}^{+0.18}$ \\
\hline . & $F 606 W_{\mathrm{R} 1}$ & $24.11 \pm 0.06$ \\
\hline$\ldots$ & $F 814 W_{\mathrm{R} 1}$ & $23.91 \pm 0.06$ \\
\hline Einstein radius of the main arc & $R_{\text {arc }}\left({ }^{\prime \prime}\right)$ & 1.4 \\
\hline Group redshift $^{a}$ & $z_{\text {grp }}$ & $0.648 \pm 0.001$ \\
\hline Group velocity dispersion ${ }^{a}$ & $\sigma_{\mathrm{grp}}^{\text {spec }}$ & $770 \pm 170 \mathrm{~km} \mathrm{~s}^{-1}$ \\
\hline $\mathrm{X}$-ray temperature ${ }^{a}$ & $k T$ & $2.0_{-0.6}^{+1.0} \mathrm{keV}$ \\
\hline$\sigma$ from $\sigma-T_{\mathrm{X}}$ relation $^{a}$ & $\sigma_{\mathrm{grp}}^{\mathrm{X}}$ & $520 \pm 120 \mathrm{~km} \mathrm{~s}^{-1}$ \\
\hline
\end{tabular}

environment on the dark matter haloes of galaxies falling into overdense environment. The system is unusual for several reasons. First, it is producing multiple images of two sources at different redshifts, similarly to the double Einstein Ring discovered by Gavazzi et al. (2008). Second, the system is located near a well-constrained group environment, and we were able to gather a large spectrophotometric set of information for both the lensing galaxy and its environment. Using this wealth of information, we are able to break some of the classic degeneracies of lensing studies. This allows us to infer the relative distribution of light and dark matter in the lens galaxy out to 1.5 effective radii of the lens galaxy, and probe the effect of the nearby group potential.

The paper is organised as follows. After presenting the data set available for SL2SJ02176-0513 in Sect. 2, we measure the optical properties of the lens galaxy (including stellar mass) in Sect. 3, and then present our lens modelling in Sect. 4. In Sect. 6, we discuss the main conclusions from the modelling and future possible improvements.

Unless otherwise stated we assume a concordance cosmology with $H_{0}=70 h_{70} \mathrm{~km} \mathrm{~s}^{-1} \mathrm{Mpc}^{-1}, \Omega_{\mathrm{m}}=0.3$ and $\Omega_{\Lambda}=0.7$. At the redshift of the lens SL2SJ02176-0513 $z_{\text {lens }}=0.6459$, $1^{\prime \prime} \sim 6.9 \mathrm{kpc}$. All magnitudes are expressed in the AB system.

\section{Observational data}

In this section we present the ensemble of available data. Table 1 summarises the main quantities of interest for this work. The ellipticity is defined as $\epsilon=\left(a^{2}-b^{2}\right) /\left(a^{2}+b^{2}\right)$. Masses are given with their photometric errors. References: ${ }^{a}$ Geach et al. (2007). 


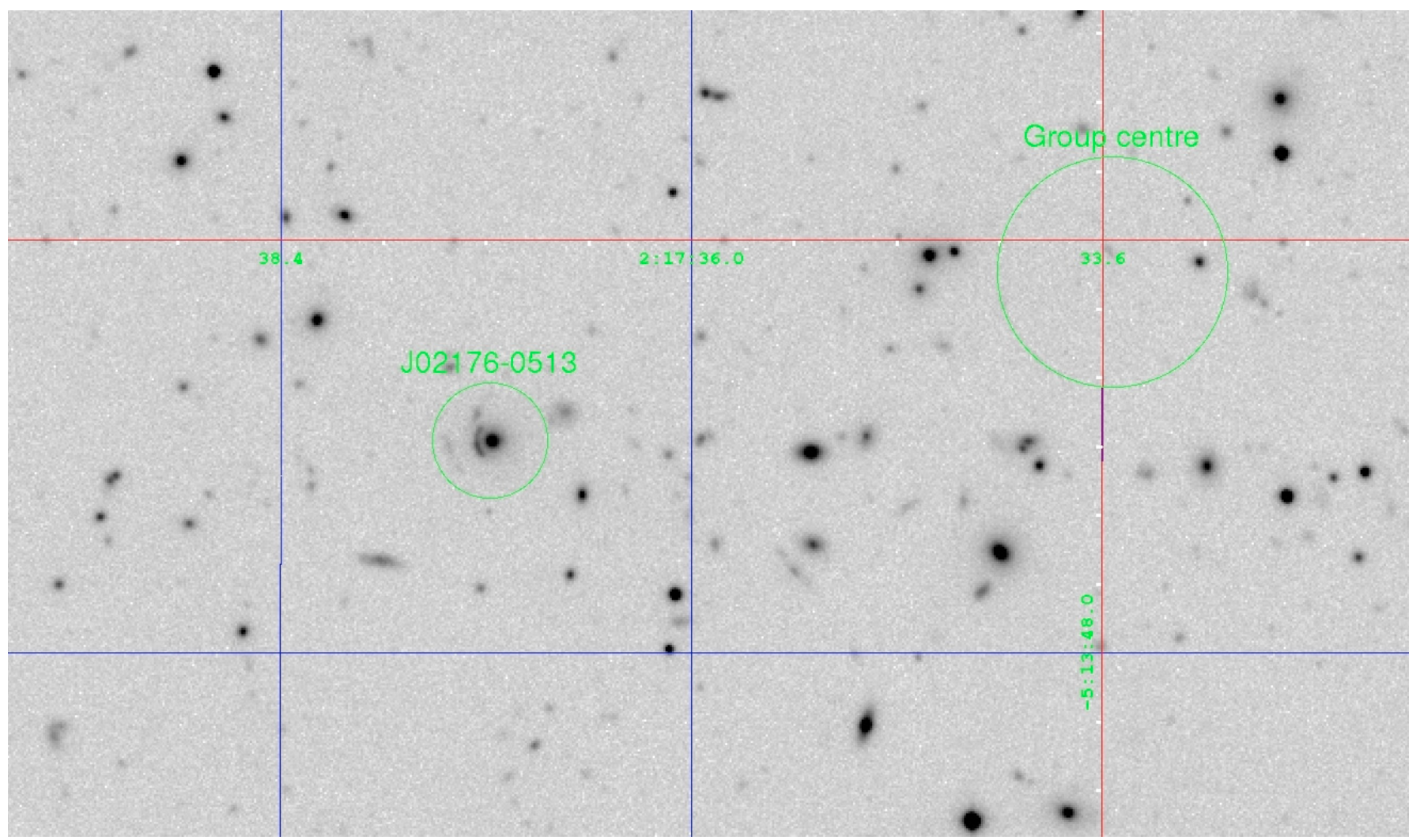

Fig. 1. Wide field CFHT image of SL2SJ02176-0513 (irg "inverted" composite image). The big 10" radius circle shows the location of the nearby galaxy group studied by Geach et al. (2007).

Note that errors on photometry are dominated by systematic calibration uncertainties.

\subsection{SL2SJ02176-0513 in the CFHTLS imaging data}

The SL2S detection strategy of lensing events was introduced by Cabanac et al. (2007). Details of the ringfinder detection algorithm, as well as the full sample of gravitational Einstein rings, will be presented in a forthcoming paper (Gavazzi et al. 2009, in prep.). As part of this effort, the gravitational lens SL2SJ021760513 was detected as a promising galaxy-scale lens candidate. Subsequent high-resolution follow-up imaging and spectroscopy have been obtained since. SL2SJ02176-0513 belongs to the W1 field in the wide part of the CFHTLS survey. Currently, subarcsecond seeing imaging is available in the Megacam bands $u^{*}, g^{\prime}, r^{\prime}, i^{\prime}$ and $z^{\prime}$. A large-scale colour image of SL2SJ021760513 is shown in Fig. 1. The coordinates of the elliptical lens on the CFHTLS image is $\alpha_{\mathrm{J} 2000}=02: 17: 37.136, \delta_{\mathrm{J} 2000}=$ $-05: 13: 29.41$.

\subsection{Radio and $X$-ray properties}

Simpson et al. (2006) found this object to be a radio source (VLA J2000 02:17:37.21 -05:13:27.96; SXD J2000 02:17:37.21 -05:13:28.0). Its environment was investigated by Geach et al. (2007). The lens galaxy inhabits a massive galaxy group (or equivalently a low mass galaxy cluster) at $z_{\text {grp }}=$ $0.648 \pm 0.001$, with an optical spectroscopic velocity dispersion $\sigma_{\text {grp }}^{\text {spec }}=770 \pm 170 \mathrm{~km} \mathrm{~s}^{-1}$. They also quote a group X-ray temperature of $k T_{\mathrm{X}}=1.97_{-0.59}^{+1.00} \mathrm{keV}$ and a group X-ray luminosity of $L_{\mathrm{X}},[0.3,10 \mathrm{keV}]=1.79_{-0.83}^{+0.29} \times 10^{43} \mathrm{erg} / \mathrm{s}$. Geach et al. (2007) give the XMM map that allows us to set the centroid of the group-extended X-ray emission to be $\alpha_{\mathrm{J} 2000}=02: 17: 33.534$, $\delta_{\mathrm{J} 2000}=-05: 13: 14.93$, with an accuracy of $\pm 10^{\prime \prime}:$ the group centre is therefore $56 \pm 10^{\prime \prime}$ from SL2SJ02176-0513. The $L_{X}-\sigma$ relation for groups of galaxies (see Fig. 5 in Geach et al. 2007) shows that the measured group velocity dispersion is somewhat higher than one would expect from its X-ray luminosity: the $L_{\mathrm{X}}-\sigma$ relation predicts $\sigma_{\mathrm{grp}}^{\mathrm{X}}=520 \pm 120 \mathrm{~km} \mathrm{~s}^{-1}$. We notice in Fig. 6 of Geach et al. (2007) that the positive and negative radial velocities of the group members do not seem to be distributed randomly across the group, but are rather distributed in a symmetric way on each side of the X-ray group centre. This may be a hint that the group is not relaxed, hence that the measured spectroscopic velocity dispersion might best be taken as an upper limit on the depth of the potential well. We note that Geach et al. (2007) also identified the lensing nature of the system and measured both the lens and doubly-imaged background source redshifts, finding $z_{\text {lens }}=0.646$ and $z_{\text {arc }}=1.847$.

\subsection{HST observations}

SL2SJ02176-0513 was observed with the Hubble Space Telescope Advanced Camera for Surveys (HST/ACS) in the $F 814 W$ and $F 606 W$ bands, as part of our SL2S follow-up snapshot programme (SNAP10876, PI Kneib). A standard reduction was done with multidrizzle and we used LACosmic for cosmic ray removal. This method happened to be more efficient in the $F 814 W$ band image, which is made of $2 \times 400$ s exposures, but gave satisfying results in the single $1 \times 400$ s F606W exposure. Figure 2 shows an image of SL2SJ02176-0513 in this latter band, with lensed features labelled (see Sect. 4.1). 


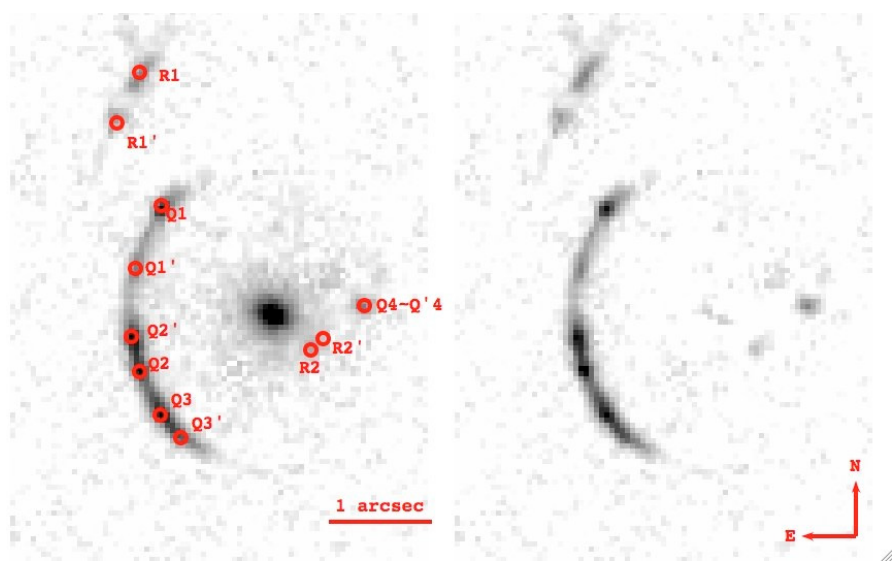

Fig. 2. HST image (F606W band) of SL2SJ02176-0513. The lens redshift is $z_{\text {lens }}=0.6459$. The main conjugate points Q1, Q2, Q3, Q4 forming the cusp arc (at redshift $z_{\text {arc }}=1.847$ ) as well as the additional double-image system R1-R2 are shown.

\subsection{Keck I spectroscopy}

We obtained a spectrum of SL2SJ02176-0513 with LRIS spectrograph on the Keck I telescope during the night of December 23rd 2006. Observations were made in long slit mode (1".5 wide) and oriented East-West. The spectrum therefore presented two parallel tracks that allowed us to measure at the same time the lens galaxy redshift $z_{\text {lens }}=0.6459 \pm 0.0003$ (based on the typical absorption features like CaII $\mathrm{H} \& \mathrm{~K}, \mathrm{G}$ band, Mgb) and the lensed arc redshift $z_{\text {arc }}=1.847$, using the strong emission lines Ly $\alpha$, CIV, HeII, OIII], and CIII]. Most of them are typical of a narrow line type II AGN (e.g. Steidel et al. 2002). This suggests that the radio source and associated active nucleus, identified by Simpson et al. (2006) and Geach et al. (2007), might actually not be at the group redshift $z_{\text {grp }} \simeq z_{\text {lens }}$ but rather at redshift 1.847. High resolution VLBI imaging would be able to demonstrate whether the radio emission is consistent with being lensed by the foreground galaxy.

\section{Lens galaxy properties}

The HST imaging allows us to make precise shape measurements of the lens galaxy optical surface brightness. Using the galfit facility (Peng et al. 2002), we found that the lens galaxy is well fit by a Sérsic surface mass density profile (assuming constant stellar mass-to-light ratio) of the form

$\Sigma(R)=\Sigma_{e} \exp \left[-b_{n}\left(\left(R / R_{\mathrm{e}}\right)^{1 / n}-1\right)\right]$,

with Sérsic index $n \simeq 5.2$ (corresponding to $b_{n} \simeq 10.07$ ) and effective radius $R_{\mathrm{e}}$. We thus measured total model magnitudes $F 606 W=20.90 \pm 0.05$ and $F 814 W=19.71 \pm 0.01$ and effective radii $R_{\mathrm{e}}=0 .^{\prime} 90 \pm 0 .^{\prime} 10 \simeq 6.20 \pm 0.7 h_{70}^{-1} \mathrm{kpc}$ in the $F 814 W$ band. In addition, galfit yielded the axis ratio $q_{*}=b / a=$ $0.87 \pm 0.05$, such that the ellipticity $\epsilon_{*}=0.14 \pm 0.06$ where $\epsilon \equiv\left(1-q^{2}\right) /\left(1+q^{2}\right)$, and the position angle $\mathrm{PA}_{*}=78 \pm 6^{\circ}$ (North to East, ccw) ${ }^{3}$. The measured flux in $F 814 \mathrm{~W}$ band was converted into an absolute rest frame $V$ band luminosity of $L_{V}=(1.36 \pm 0.08) \times 10^{11} h_{70}^{-2} L_{\odot}$. This includes corrections from Galactic extinction (Schlegel et al. 1998), the small filter shifting correction to translate $z=0.6459 F 814 W$ into a rest frame

\footnotetext{
${ }^{3}$ Note that systematic uncertainties dominate the galfit run. We use the difference between estimates in the $F 814 \mathrm{~W}$ and $F 606 \mathrm{~W}$ bands as an error estimate for both $q_{*}$ and $\mathrm{PA}_{*}$.
}

$V$ band, in nearly the same way as (Gavazzi et al. 2007) except that, here we do not apply any passive evolutionary correction to the reference redshift zero epoch. To do so, one could use the relation constrained by Fundamental Plane evolution studies (Treu et al. 2001, 2006):

$$
\frac{\mathrm{d} \log \frac{M_{*}}{L_{V}}}{\mathrm{~d} z} \simeq-0.40 \pm 0.05 \text {. }
$$

CFHT photometry within a $1^{\prime \prime}$ radius aperture around the lens centre allows us to estimate the corresponding cylindrical stellar mass. Note that we applied small $\sim 4 \%$ corrections to compensate aperture flux losses due to ground-based seeing (ranging from $1^{\prime \prime} .1$ in $u^{*}$ to $0^{\prime} .6$ in $i^{\prime}$ ). CFHT magnitudes were also corrected for Galactic extinction. Using the publicly available code hyperz (Bolzonella et al. 2000) with a Chabrier Initial Mass Function (IMF) (Chabrier 2005) and an intrinsic absorption coefficient $A_{\mathrm{V}}=0$ typical for nearby early-type galaxies, we obtain a stellar mass of $M_{*}\left(<1^{\prime \prime}\right)=1.4_{-0.2}^{+0.6} \times 10^{11} M_{\odot}$. Using a Salpeter IMF and/or a non-zero $A_{\mathrm{V}}$ up to 0.3 (unlikely for such a massive early-type galaxy), the inferred mass reaches $M_{*}\left(<1^{\prime \prime}\right) \simeq 1.7 \times 10^{11} M_{\odot}$. It results that, although dominated by stellar evolution systematics, the stellar mass of the lens galaxy within a one-arcsec radius is likely in the range $1.2-2.3 \times$ $10^{11} M_{\odot}$. Using the Sérsic profile measured on HST data, we can extrapolate this aperture mass measurement and estimate a total "photometric" stellar mass $M_{*}=2.1-4.1 \times 10^{11} M_{\odot}$. This value corresponds to a $V$-band stellar Mass-to-light ratio $M_{*} / L_{V}=1.5-3.0(M / L)_{\odot}$ at $z_{\text {lens }}=0.646$. Accounting for luminosity evolution as described above (Eq. (2)), we get an equivalent zero-redshift $M_{*} / L_{V}=2.8-5.5(M / L)_{\odot}$, which is consistent with, although slightly lower than, local estimates (Gerhard et al. 2001; Trujillo et al. 2004), but is in good agreement with the strong+weak gravitational lensing constraints of Gavazzi et al. (2007), who found an average $M_{*} / L_{V}=3.84 \pm 0.40$ in SLACS lens galaxies, once they were evolved to redshift zero using Eq. (2).

\section{Strong lensing analysis}

In this section, we present our strong lensing analysis. It was performed using the latest version of the lenstool code (Jullo et al. 2007), which uses a Markov Chain Monte Carlo sampler to characterise the posterior PDF for the model parameters.

\subsection{Identification of multiple images}

lenstool relies on the identification of conjugate multiple images of the same background source. For a source imaged $N$ times, since the unlensed source position is unknown, the location of each of these points in the image plane results in $2(N-1)$ independent constraints. When a source is structured in such a way that multiple sub-components (surface brightness peaks) can be identified, one can significantly increase the amount of information provided by the lensing configuration.

For the HST/ACS images we are considering here, we find the typical astrometric uncertainties on the positions of identified conjugate image features to be $0{ }^{\prime} 03$. These constraints are summarised in Table 2. Units are arcseconds West and North relative to the lens galaxy centre. Uncertainties are set to a typical value of $0{ }^{\prime}$ '03. Column "\# const" gives the contribution to the total amount of constraints supplied to the lenstool model. The subsections below detail the way in which multiple images are identified. 
Table 2. Positions of multiply-imaged, conjugate image features used in the lenstool analysis.

\begin{tabular}{lccccc}
\hline \hline & Image 1 & Image 2 & Image 3 & Image 4 & \# const. \\
\hline$Q$ & $-1.06,1.03$ & $-1.29,-0.53$ & $-1.06,-0.98$ & $0.88,0.08$ & 6 \\
$Q^{\prime}$ & $-1.33,0.41$ & $-1.34,-0.25$ & $-0.90,-1.18$ & $0.88,0.08$ & 6 \\
$R$ & $-1.28,2.31$ & $0.39,-0.33$ & & & 2 \\
$R^{\prime}$ & $-1.50,1.89$ & $0.45,-0.25$ & & & 2 \\
total & & & & & 16 \\
\hline
\end{tabular}

The HST/ACS image in Fig. 2 clearly reveals that SL2SJ02176-0513 is causing the formation of a tangential arc (Q1-Q2-Q3, in a typical cusp configuration) along with its small and faint counter-image (Q4) on the opposite side of the lens, about twice as close to the lens centre. The arc is bright $\left(F 606 W_{\text {arc }}=22.04 \pm 0.16\right.$ and $\left.F 814 W_{\text {arc }}=22.19 \pm 0.13\right) . \mathrm{A}$ rough estimate of its length-to-width ratio $r$ yields $r \simeq 8$, which means that the unlensed source magnitudes are $\sim 2.3$ mag dimmer. The arc has a well-extended surface brightness distribution resulting from the merging of 3 conjugate multiple images separated by the critical line. The source thus has to lie across the caustic with parts of the source pointing outside the tangential cusp. Those parts of the source will only be imaged 3 times, whereas the others will be imaged 5 times ${ }^{4}$. This configuration makes the identification of conjugate images of substructures along the arc quite difficult ex nihilo.

We approach this problem in two stages. In the first stage, we perform a modelling using the most readily identified (and therefore most robust) conjugate points in the arc and its counterimage only. This provides a satisfactory model which we then use for testing further the more difficult conjugate images along the arc, finally reaching the astrometric precision of 0 ' $^{\prime} 03$ in the predicted image points.

An interesting feature of SL2SJ02176-0513 is that a highlyelongated arclet shape is present at about 2'.5 North-East of the lens galaxy (labelled R1 in Fig. 2). A photometric redshift of this system $z_{\mathrm{dbl}}=2.90_{-0.24}^{+0.18}$ was obtained from the CFHTLS photometry with hyperz. The lens modelling of the tangential system Q1-Q2-Q3-Q4 predicts that R1, at that redshift, should be multiply-imaged. Indeed, we do observe a demagnified counterimage R2 (clearly in the $F 606 \mathrm{~W}$ image, and at slightly lower signal-to-noise in the $F 814 W$ image). R2 is unfortunately too faint and buried in the light of the lens galaxy for an accurate confirmation of its photometric redshift. However, in the HST imaging we were able to measure photometry for R2 and R1, leading to colour indices $(F 814 W-F 606 W)_{\mathrm{R} 1}=0.19 \pm 0.08$ and $(F 814 W-F 606 W)_{\mathrm{R} 2}=-0.15 \pm 0.85$ that are at least consistent with one another, although this constraint is relatively poor due to the extreme faintness of $\mathrm{R} 2(F 606 W=27.15 \pm 0.54$; $\mathrm{R} 1$ is much brighter, $F 606 W=24.11 \pm 0.06)$.

We can also estimate the "geometric redshift" of the R1-R2 source by including it in the lens modelling. We identify two secondary conjugate brightness peaks $\mathrm{R}^{\prime} 1-\mathrm{R}^{\prime} 2$, and use these with $\mathrm{R} 1-\mathrm{R} 2$ to provide 4 new astrometric constraints. We infer the value of $z_{\mathrm{dbl}}$ to be very close to the photometric value.

Throughout the rest of this work, we fix the redshift $z_{\mathrm{dbl}}$ of the double-image system to its photometric redshift estimate $z_{\mathrm{dbl}}=2.9$, noting that the uncertainty on this value is small enough for the corresponding error in the $D_{\mathrm{ls}} / D_{\mathrm{s}}$ distance ratio to be negligible. In addition one might expect a third pair of

\footnotetext{
${ }^{4}$ In practice, this odd number of conjugate images reduces to 2 and 4 multiple images respectively because one of the images (the central one) is highly demagnified and almost never observed.
}

images R3-R'3 of opposite parity to R2-R'2 with respect to the radial critical line - if it exists. These demagnified counterimages are predicted by our lens model to form well underneath the elliptical galaxy, thus explaining why we do not observe them. This non-detection prevents us from accurately fixing the location of the radial critical line, but the relative distances between R1-R'1 and R2-R'2 still allow us to extend the range of radial constraints.

\subsection{Model assumptions}

We now describe our mass model for SL2SJ02176-0513 and its group environment. The lens itself is modelled with two components describing the stars in the lens galaxy, and the dark matter (DM) halo in which they sit. For the surface density of the former, we assume a Sérsic profile for which most model parameters (effective radius $R_{e}$, index $n$, ellipticity and orientation) are fixed at the values determined from the galfit model of the HST images. Only the total stellar mass $M_{*}$ is taken as a free parameter. Given the total rest-frame $\mathrm{V}$ band luminosity $L_{V}=(1.36 \pm 0.08) \times 10^{11} h_{70}^{-2} L_{\odot}$, one can readily translate this parameter into a stellar mass-to-light ratio.

The dark matter halo surrounding the lens galaxy is modelled as a pseudo isothermal mass distribution (PIEMD, Kassiola \& Kovner 1993; Limousin et al. 2005). We also considered a NFW profile (Navarro et al. 1997b) as suggested by $N$-body simulations, and found that none of our results change significantly either in terms of goodness-of-fit or regarding the main qualitative trends described in the results section below. We fix the centre of the halo to that of the galaxy light distribution, building on well-established results from previous galaxy-scale strong lensing studies (e.g. Yoo et al. 2006). (Moreover, when the centroid was freed in our modelling, we found that the centre of the DM halo has to be $\lesssim 0$ ' 1 from the centre of light.) The orientation $\mathrm{PA}_{\mathrm{dm}}$, ellipticity $\epsilon_{\mathrm{dm}}$, and velocity dispersion parameter $\sigma_{\mathrm{dm}}$ of the DM halo are let free with broad uniform priors $0 \leq \epsilon_{\mathrm{dm}} \leq 0.6$, $0 \leq \mathrm{PA}_{\mathrm{dm}} \leq 180^{\circ}$, and $\sigma_{\mathrm{dm}} \leq 450 \mathrm{~km} \mathrm{~s}^{-1}$. We also allow the possibility of a finite core radius in the range $0<r_{\mathrm{c}, \mathrm{dm}}<1^{\prime \prime}$. 5 . Due to the relatively small projected distance to the cluster centre $(\simeq 400 \mathrm{kpc})$, the galaxy may have a large part of its DM halo stripped. Lacking observational constraints, we set the truncation radius $r_{\text {cut }}$ to $60 \mathrm{kpc}$, an average value motivated by simulation and galaxy-galaxy lensing results in similarly high density, cluster environments (Natarajan et al. 2002b,c; Limousin et al. 2007a).

Finally, the host halo of the galaxy group to which SL2SJ02176-0513 belongs will have some impact on the lensing properties of the main lens galaxy. To first order, the perturbing effect of the group can be characterised by an external shear, oriented tangentially relative to the group centre. A substantial amount of extra convergence should also be supplied by this nearby group DM halo. Since we know where the group is (from the X-ray observations, Sect. 2), we thus decide to model the group by a Singular Isothermal Sphere (SIS) with velocity dispersion $\sigma_{\text {grp }}$. This model not only predicts the external shear and its associated convergence, but also naturally accounts for any higher order deflections at the lens position. Typically, if the group velocity dispersion suggested by the kinematics of group member galaxies or the intra-cluster gas temperature is correct $\left(\sigma_{\text {grp }} \sim 630 \mathrm{~km} \mathrm{~s}^{-1}\right)$, one would expect the group to produce external shear and convergence of $\left|\gamma_{\text {ext }}\right| \sim \kappa_{\text {ext }} \simeq 0.05$ at the location of the lens. We thus include $\sigma_{\text {grp }}$ as the last free parameter of the model, assigning a uniform prior $0 \leq \sigma_{\mathrm{grp}} \leq 900 \mathrm{~km} \mathrm{~s}^{-1}$ to be 
able to compare the group mass estimate with the independent $\mathrm{X}$-ray and spectroscopic values.

Altogether we have a total of 6 free parameters in our lens model. However, instead of further considering the raw model parameters above, we introduce the following secondary transformed quantities, more relevant for the discussion below: the group velocity dispersion $\sigma_{\text {grp }}$, the total stellar mass of the lens galaxy $M_{*}$, the dark matter fraction enclosed within the cylinder defined by the effective radius $f_{\mathrm{dm}}\left(<R_{\mathrm{e}}\right)$, the difference in orientations of the dark matter halo and the stellar component $\triangle \mathrm{PA}$ (for which the errors in the light orientation $\mathrm{PA}_{*}=78 \pm 6$ are added in quadrature to the $\mathrm{PA}_{\mathrm{dm}}$ estimate), and the ellipticity of the dark matter halo $\epsilon_{\mathrm{dm}}$.

\subsection{Inclusion of more informative priors}

We consider two working hypotheses for modelling SL2SJ02176-0513. As our primary and default strategy, we adopt the most informative prior - based on the stellar mass derived from the $u^{*} g^{\prime} r^{\prime} i^{\prime} z^{\prime}$ aperture photometry - i.e. a uniform prior $2.1 \leq M_{*} \leq 4.1 \times 10^{11} M_{\odot}$ (hereafter referred to as $M_{*}$ prior). As a sanity check and to investigate the effects of the informative prior, we also consider the broad uniform priors on the primary parameters $\sigma_{\mathrm{grp}}, \epsilon_{\mathrm{dm}}, \mathrm{PA}_{\mathrm{dm}}, r_{\mathrm{c}, \mathrm{dm}}, \sigma_{\mathrm{dm}}$ and $M_{*}$ introduced above. This case will be referred to as broad prior. Although we refer primarily to the parameters inferred from the $M_{*}$ prior, we also report the parameters inferred from the broad prior for completeness. As a further check, we investigated the assumption that the dark matter halo and the stars are aligned on the scales probed by the strong lensing features $(6 \lesssim R \lesssim 12 \mathrm{kpc}$ ). It turned out that this prior provides virtually the same constraints as the broad prior case, so we do not discuss it, for the sake of conciseness.

\section{Results}

The lens modelling yields good fits to the positions of all the multiple images. The recovered model parameters, along with their $68 \%$ confidence level uncertainties and goodness-of-fit quantified in terms of $\chi^{2}$ per degree of freedom - are listed in Table 3. Reported values are the median and the $16 \%$ and $84 \%$ quantiles around it from the marginalised one-dimensional posterior distributions. Results from the two priors cases are listed for comparison. Derived constraints on additional secondary parameters are also listed (slopes are defined in Sect. 5.2). In Fig. 3, we show the excellent matching between the observed positions of the multiple images (from Table 2) and the ones predicted by the best-fit model. We stress here that the combination of medians of marginalised one-dimensional posterior distribution for each parameter (as listed in Table 3) does not necessarily correspond to the mode of the overall posterior probability distribution that we nominate as "best fit model" and approximate with the highest likelihood MCMC sample.

\subsection{Model parameters}

We show the marginalised posterior distributions for both the $M_{*}$ and broad prior cases in Fig. 4. We illustrate pair-wise degeneracies between the most relevant parameters by plotting the complete (except for the unconstrained core radius dimension) set of 2D marginalised posterior PDFs. One-dimensional marginalised posterior distributions are also shown in the diagonal panels with overlaid, in blue, the implicit prior that we
Table 3. Summary of the recovered model parameters. Chi-squared values refer to the "best-fit" model.

\begin{tabular}{ccc}
\hline \hline & $\begin{array}{c}M_{*} \text { prior' } \\
\text { from photometry }\end{array}$ & broad prior \\
\hline constraints & 16 & 16 \\
free parameters & 6 & 6 \\
$\chi^{2}$ & 3.1 & 3.5 \\
$\chi^{2} /$ d.o.f. & 0.31 & 0.35 \\
\hline$M_{*}\left(10^{11} M_{\odot}\right)$ & $3.4_{-0.7}^{+0.5}$ & $6.6_{-2.1}^{+0.8}$ \\
$\epsilon_{\mathrm{dm}}$ & $0.16_{-0.12}^{+0.20}$ & $0.27_{-0.19}^{+0.22}$ \\
$\Delta \mathrm{PA}_{\mathrm{dm}}$ & $20_{-41}^{+16}$ & $23_{-62}^{+42}$ \\
$r_{\mathrm{c}, \mathrm{dm}}$ & $0.48_{-0.33}^{+0.52}$ & $0.72_{-0.49}^{+0.52}$ \\
$\sigma_{\mathrm{dm}}\left(\mathrm{km} \mathrm{s}{ }^{-1}\right)$ & $218_{-28}^{+43}$ & $106_{-72}^{+85}$ \\
$\sigma_{\text {grp }}\left(\mathrm{km} \mathrm{s}{ }^{-1}\right)$ & $550_{-240}^{+130}$ & $690_{-145}^{+90}$ \\
\hline Total mass within $R_{\text {Ein }}\left(10^{11} M_{\odot}\right)$ & $5.8_{-0.4}^{+0.3}$ & $7.8_{-1.1}^{+0.5}$ \\
Dark Matter fraction $f_{\mathrm{dm}}\left(<R_{\mathrm{e}}\right)$ & $0.41_{-0.06}^{+0.09}$ & $0.06_{-0.06}^{+0.18}$ \\
Group contribution $\left|\gamma_{\mathrm{ext}}\right| \sim \kappa_{\mathrm{ext}}$ & $0.039_{-0.026}^{+0.021}$ & $0.061_{-0.023}^{+0.017}$ \\
Slope $\alpha$ & $-1.03_{-0.16}^{+0.22}$ & $-1.45_{-0.16}^{+0.30}$ \\
Slope $\alpha^{\prime}$ & $-1.11_{-0.18}^{+0.27}$ & $-1.68_{-0.22}^{+0.42}$ \\
\hline
\end{tabular}

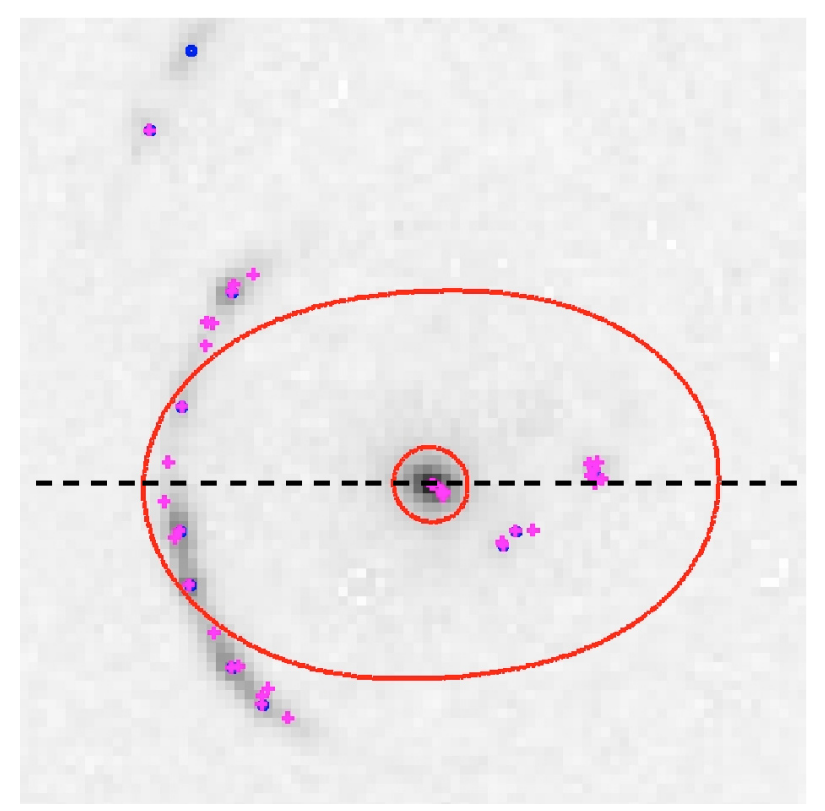

Fig. 3. Illustration of the accuracy of the best fit model. Small blue circles are input data while red crosses are model-predicted image positions after optimisation. Critical curves are shown as red solid lines. For reference, the distance of the galaxy centre to the main arc is about 1". 4 .

assumed for the derived parameters when making the broad prior assumptions about the primary parameters. This shows that - by construction - our apparently uninformative broad prior model assumptions will tend to maximise the mass component that is in the form of stars.

We note the following about our inferences:

- the misalignment between mass and light $\Delta \mathrm{PA}=20_{-41}^{+16} \mathrm{deg}$ is preferred by the $M_{*}$ prior. However, the same prior also prefers a rounder halo, making the misalignment of little physical significance. With the broad prior, the misalignment of the dark matter halo and the stellar component looses its statistical significance as well; 

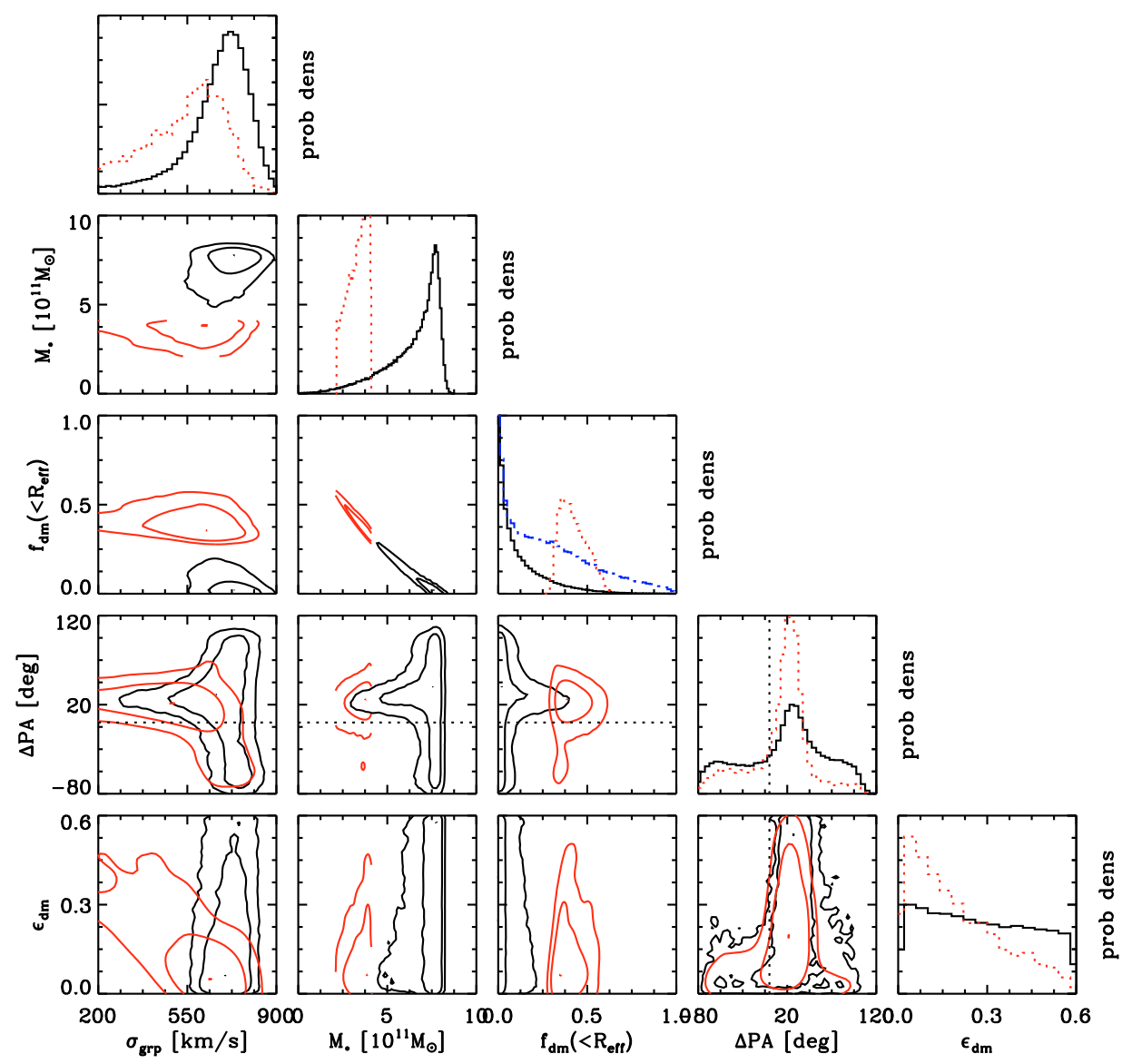

Fig. 4. Confidence regions for all model parameters, except the lens galaxy core radius, which is unconstrained. For the offdiagonal 2D plots, contours represent 1 and $2-\sigma$ confidence levels. Along the diagonal, 1D marginalised distributions are shown. Two different prior PDF assignments are considered, the basic broad prior (black) and the $M_{*}$ prior (red) based on the $\left\{u^{*} g^{\prime} r^{\prime} i^{\prime} z^{\prime}\right\}$-band CFHTLS photometry and for which stellar mass $M_{*}=2.1-4.1 \times$ $10^{11} M_{\odot}$. The dot-dashed blue curve in the $f_{\mathrm{dm}}\left(<R_{\mathrm{e}}\right)$ PDF panel represents the (unnormalised) implicit broad prior on this derived parameter.

- the lens model requires a substantial contribution from the group halo: for the $M_{*}$ prior we infer $\sigma_{\text {grp }}=550_{-240}^{+130} \mathrm{~km} \mathrm{~s}^{-1}$, which is consistent with both the velocity dispersion of galaxies in the group $\left(\sigma_{\text {grp }}^{\text {spec }}=770 \pm 170 \mathrm{~km} \mathrm{~s}^{-1}\right)$ and the prediction from the $\mathrm{X}$-ray luminosity scaling relation $\left(\sigma_{\mathrm{grp}}^{\mathrm{X}}=\right.$ $520 \pm 120 \mathrm{~km} \mathrm{~s}^{-1}$ ). We observe that the more massive the group, the less the contribution of a dark matter halo is needed on the scales probed by the Einstein ring. A less massive group halo is more in line with $M_{*}$ prior as we get $\sigma_{\text {grp }}=690_{-145}^{+90} \mathrm{~km} \mathrm{~s}^{-1}$ for the broad prior. The informative prior thus allows us to obtain tighter constraints on the mass of the group;

- the overall group contribution to the mass budget within the Einstein radius is about $4 \%$, while we find it contributes about $8-13 \%$ to the local density at the Einstein radius. The implications for the mass budget in the lensing galaxy is thus not negligible. This important issue is discussed in more detail below;

- the galaxy halo dark matter fraction inside the effective radius is highly sensitive to the choice of prior. For the $M_{*}$ prior we obtain $f_{\mathrm{dm}}\left(<R_{\mathrm{e}}\right)=0.41_{-0.06}^{+0.09}$. Assuming the broad prior would give a much lower value of $f_{\mathrm{dm}}\left(<R_{\mathrm{e}}\right)=0.06_{-0.06}^{+0.18}$. The number obtained with the informative $M_{*}$ prior is consistent with local estimates from the SLACS survey (e.g. Gavazzi et al. 2007; Bolton et al. 2008b), where typically 30\% of the projected mass inside the effective radius is found to be in the form of dark matter, independent of any assumption on the stellar populations. The broad prior yields a much smaller number, which is hard to reconcile with other observations. This lends confidence to the use of the informative $M_{*}$ prior as our primary choice;
- the core radius in the dark matter distribution is unconstrained by the data, so we do not show the posterior distribution, which is indistinguishable from the input prior.

\subsection{The mass density profile of SL2SJ02176-0513}

Thanks to the appearance in SL2SJ02176-0513 of two sets of multiple-images from two sources at different redshifts, we can expect, by comparison with the recently discovered double Einstein ring SDSSJ0946+1006 (Gavazzi et al. 2008), this lens to provide tight constraints on the inner parts of its total density profile. Gavazzi et al. (2008) show how important it is to take the multiple deflector plane ("compound lens") nature of such systems into account, since the nearest background source further bends the light rays coming from the most distant source. In the case of SL2SJ02176-0513, the two sources are much farther away from the foreground galaxy than in SDSSJ0946+1006, where the inner ring is at $z=0.609$. Furthermore, the source positions are not as closely aligned with the lens galaxy as in SDSSJ0946+1006, where the sources gave rise to almost complete Einstein Rings. Thus, the impact parameter of the most distant source in the plane of the intermediate source is much larger than for SDSSJ0946+1006 in units of the Einstein radius of the intermediate galaxy (assuming a typical size galaxy). It is therefore unnecessary to treat SL2SJ02176-0513 as a compound lens. In addition, the image configuration provides too little information to put constraints on the mass of the intermediate $z_{\text {arc }}=1.847$ galaxy, confirming that its effect is insignificant for this problem.

The nearby galaxy group will provide a substantial amount of convergence at the lens location, making the issue of the 


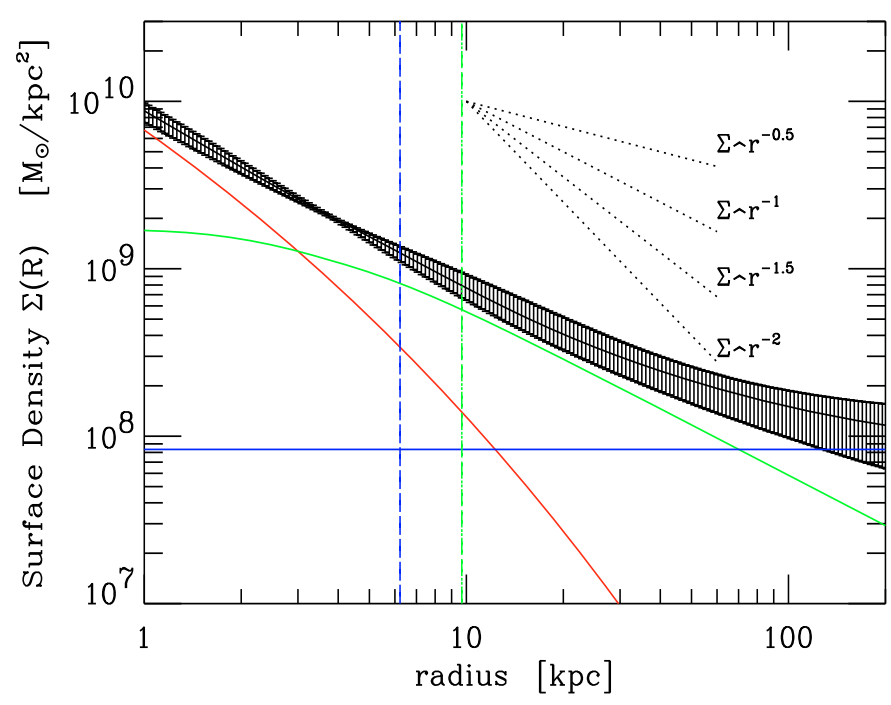

Fig. 5. Total surface mass density profile. The effective radius and the Einstein radii are shown as vertical dashed blue and dot-dashed green lines (respectively at $\sim 6.2$ and $\sim 10.3 \mathrm{kpc}$ ). The envelope around median values shows the $68 \%$ confidence interval. We show the median stellar density profile (red curve), the median DM halo profile (green) and the median effective external convergence (blue horizontal line) that successively dominate from left to right. In black is shown the total density profile - including the stars, the DM halo and the (approximately) constant convergence from the group.

projected density profile more complicated than for isolated lenses. In the previous section, we found that about $4 \%$ of the mass enclosed in the Einstein radius is supplied by the group halo. Locally, at the Einstein radius, the group halo accounts for $\sim 8 \%$ of the surface mass density. Hence the results one gets for the density profile slope of the lens galaxy strongly depend on the external convergence. This issue is illustrated well by Fig. 5, which shows the recovered surface mass density profile: at each radius the density is evaluated from the median density over all MCMC samples (the envelope shows the $68 \%$ confidence interval, estimated with the 16 and 84 percentiles). The contribution of stars is seen to dominate the density profile on small scales as expected.

Let us now quantify the density profile slope more precisely, focusing on the logarithmic projected surface mass density slope evaluated at the effective radius:

$\alpha=\left.\frac{\mathrm{d} \log \Sigma_{\mathrm{tot}}}{\mathrm{d} \log R}\right|_{R=R_{\mathrm{e}}}$.

This slope parameter would be -1 for an isothermal density profile. We can compute $\alpha$ for every MCMC sample and thus build up the posterior PDF $P(\alpha \mid$ data). We plot this distribution in the top panel of Fig. 6; again, we are showing here the total profile slope, including dark halo, stellar and group mass components.

Referring to Fig. 5, we can see that out to $\sim 4$ effective radii the total density profile is well-approximated by a single powerlaw. Figure 6 shows that this power-law index is $\alpha=-1.03_{-0.16}^{+0.22}$, consistent with the isothermal case. However, the bottom panel shows the posterior probability distribution for the slope parameter $\alpha^{\prime}$ obtained when we remove the external convergence from the mass budget. This shifts the result towards a slightly steeper density profile $\alpha^{\prime}=-1.11_{-0.18}^{+0.27}$. Constraints on $\alpha$ and $\alpha^{\prime}$ are listed in Table 3 for the broad and $M_{*}$ priors.

As in the case of the dark matter fraction $f_{\mathrm{dm}}\left(<R_{\mathrm{e}}\right)$, the constraints we infer on these derived (secondary) parameters need to

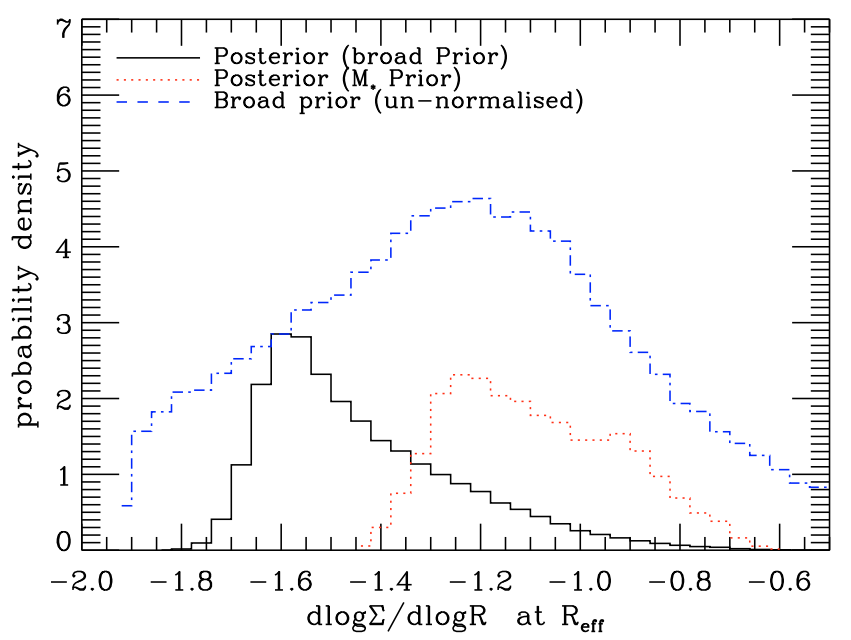

Fig. 6. Probability distribution for the logarithmic surface density slope $\alpha$ at the effective radius location for the total (stellar+DM+group convergence) density profile. The colour coding for the curves showing posterior probability distribution with different priors is the same as in Fig. 4.

be compared to the input priors we assumed. The un-normalised implicit prior probability distributions for $\alpha$ shown in Fig. 6 turns out to be fairly uniform, with only a broad peak close to -1 arising from our use of the pseudo-isothermal model DM halo (the high Sersic index stellar mass profile favours slightly steeperthan-isothermal total density slopes at $R_{\mathrm{e}}$ ).

The difference between the results obtained with the two priors can be summarised as:

- the steep density slopes are mostly driven by the high predicted stellar mass with the broad prior;

- a lower stellar mass, as favoured by the informative $M_{*}$ prior, implies density slopes that are closer to isothermal with $\alpha=$ $-1.03_{-0.16}^{+0.22}$ and $\alpha^{\prime}=-1.11_{-0.18}^{+0.27}$;

- the slopes $\alpha$ and $\alpha^{\prime}$ get closer to one another, reflecting the reduction in the contribution to the mass budget from the nearby group when the stellar mass is lowered (and the more extended DM halo is increased in mass to compensate).

\subsection{The external field - mass profile degeneracy}

Even with the two multiply-imaged systems at two different redshifts as we have here, gravitational lensing alone cannot tell us both the density profile slope and how much mass is in the form of a uniform external mass density. However, the degeneracy is broken here, via the constraints we have placed on the associated external shear provided by the group halo, the assumption that the group halo has an isothermal mass distribution, and the functional form assumed for the mass density profile of the lens galaxy (because the two multiply-imaged systems are not exactly at the same projected location in the sky). For example, by making these assumptions, the external convergence and shear strength are fixed to be equal.

As we show in Appendix A, one could also break this degeneracy with a measurement of the stellar velocity dispersion of the lensing galaxy, which would allow us to infer both the density slope and external convergence at once. We postpone this analysis for future work. We note that the combination of stellar kinematics of the lens galaxy in a single aperture and strong lensing constraints coming from a single multiply-imaged system 
(e.g. Miralda-Escudé 1995; Sand et al. 2004; Treu \& Koopmans 2004; Koopmans 2006; Koopmans et al. 2006; Czoske et al. 2008) cannot break the degeneracy between both density slope and external convergence by itself, so additional information is necessary. This could include, for example, spatially resolved stellar kinematics, information on the external mass distribution, or multiple lens planes, together with assumptions on the form of the mass distribution as in the case presented here.

So far the effect of environment has been carried by the modulus of the shear as parameterised by the mass of the nearby group of galaxies since the location of this latter was fixed by $\mathrm{X}$-ray observations. We attempted to investigate the effect of a possible miscentering. Distance and group mass are completely degenerate through the shear modulus so there is no need to study the effect of releasing the distance between SL2SJ021760513 and the group centre along the observed direction. We did, however, consider various locations of the group centre along a circle while keeping the mass and distance fixed. This procedure is similar to releasing the orientation of the external shear. By doing so, we checked that no noticeable effect related to the lens monopole parameters is significantly changed, neither is the best fit $\chi^{2}$ or Bayesian evidence. Obviously, the orientation of the dark matter halo $\mathrm{PA}_{\mathrm{dm}}$ and its associated ellipticity $\epsilon_{\mathrm{dm}}$ are changed to preserve the orientation and elongation of the overall potential. This is somewhat at odds with earlier results of Narasimha et al. (1984), who noticed a degeneracy between mass profile and external shear on the double QSO 0957+561. This can be explained by the fact that too few constraints (even with time delays) on the azimuthal symmetry of the mass distribution are supplied by double QSOs (e.g. Keeton et al. 1997; Kochanek 2002, and references therein). We thus conclude that lensing constraints on SL2SJ02176-0513 are able to break massellipticity degeneracies that perplex most lens modellings of doubly imaged sources.

\section{Discussion and conclusion}

In this paper we have attempted to produce a detailed lens modelling of one of the most complete systems in the growing "SL2S" sample of strong lensing events in the CFHT Legacy Survey. SL2SJ02176-0513 is a partial Einstein ring in the outskirts of an X-ray-emitting group of galaxies. The observational dataset available for SL2SJ02176-0513 is quite remarkable, consisting of deep $u^{*} g^{\prime} r^{\prime} i^{\prime} z^{\prime}$ CFHTLS photometry, high-resolution data from HST/ACS snapshot imaging, a published XMM X-ray map, the galaxy velocity dispersion of the host group (based on 18 radial velocities; Geach et al. 2007), and a spectroscopic measurement of the deflector and source redshifts from Keck/LRIS.

In addition, the system exhibits an extra double-image system, which we have determined lies at higher redshift than the initially detected arc/counter-arc system. This system gives an important additional handle on the radial mass distribution. To our knowledge, SDSSJ0946+1006 (Gavazzi et al. 2008) and SL2SJ02176-0513 constitute the only two known galaxy-scale systems with multiply-imaged sources at different redshifts.

The influence of the nearby group on the lens configuration is detected as an external shear that accounts for the slightly misaligned overall potential and stellar component. The lens model including a prior on the stellar mass from the CFHTLS photometry seems to favour a massive group with velocity dispersion $\sigma_{\text {grp }}=550_{-240}^{+130} \mathrm{~km} \mathrm{~s}^{-1}$, consistent with X-ray luminosity-predicted (Geach et al. 2007). The group environment contribution is somewhat degenerate with the mass of the dark matter halo of the lens galaxy: the higher the group mass, the lower the dark matter fraction in the deflector galaxy. Similarly, if the shear supplied by the group is reduced, the galaxy dark matter halo and the stellar component must be preferentially misaligned.

Despite these degeneracies between model parameters, the total radial projected mass profile is well-constrained by the double source-plane nature of SL2SJ02176-0513. However, we have shown that the slope of the galaxy stellar-plus-dark matter mass density profile at the effective radius differs according to whether the convergence supplied by the group is taken into account in the mass budget or not. The effect the group has on the apparent density profile is to make it shallower by $8 \%$ (from $\alpha^{\prime}=-1.11$ without the group contribution to $\alpha=-1.03$ with the group contribution). Again, this relatively steeper than isothermal profile is consistent with the lens PG1115+080 in the environment of a group (Treu \& Koopmans 2002). Statistical analyses of SLACS lenses also show that they tend to have slightly steeper density profiles when found in denser environments (Auger 2008) or, more precisely, when they are satellite of massive haloes (Treu et al. 2008). This latter result is also supported by simulations of Dobke et al. (2007) and Limousin et al. (2007b).

As mentioned in the Appendix, a velocity dispersion measurement of the stars in the central galaxy would help to break the remaining degeneracy in this system, tightening the constraints on the galaxy density profile and the group mass.

We are currently pursuing this observational effort, and are now working on the spectroscopic determination of the velocity dispersion of stars in the lensing galaxy. Likewise, the continuing growth of the SL2S sample will allow us to tackle the problem of understanding massive galaxy mass distributions with better statistics.

Acknowledgements. This paper is based on observations made with the NASA/ESA Hubble Space Telescope, obtained at the Space Telescope Science Institute, which is operated by the Association of Universities for Research in Astronomy, Inc., under NASA contract NAS 5-26555. These observations are associated with programs \#10876 and \#11289. Support for programs \#10876 and \#11289 was provided by NASA through a grant from the Space Telescope Science Institute. The authors are thankful to the CFHTLS members and the Terapix team for their excellent work in reducing and distributing data to the community. We thank Matthew Auger for insightful conversations. Part of this project is done under the support of National Natural Science Foundation of China Nos. 10878003, 10778752, 973Program No. 2007CB815402, Shanghai Foundation Nos. 08240514100, 07dz22020, and the Leading Academic Discipline Project of Shanghai Normal University (08DZL805). Part of this work was supported by the Agence Nationale de la Recherche (ANR) and the Centre National des Études Spatiales (CNES). M.L. acknowledges the ANR and CNES for its support. The Dark Cosmology centre is funded by the Danish National Reasearch Fundation. P.J.M. acknowledges the TABASGO foundation for support in the form of a research fellowship. T.T. acknowledges support from the National Science Foundation through CAREER award NSF-0642621, by the Sloan Foundation through a Sloan Research Fellowship, and by the Packard Foundation through a Packard Fellowship.

\section{Appendix A: Constraints on density slope and external convergence with three aperture masses}

In this appendix, we demonstrate that the combination of two multiply-imaged sources at different redshifts, which define two projected aperture mass measurements, and an additional measurement of a small-scale stellar velocity dispersion, which can be approximated as a three-dimensional aperture mass estimate, allow us to constrain simultaneously the density profile slope parameter and the external convergence due to mass lying both along the line of sight and in the lens plane outside 
the lens galaxy (but that does not affect the lens galaxy's stellar dynamics).

Let us define a density profile of the form

$$
\begin{aligned}
\rho(r) & =\rho_{0}\left(r / r_{0}\right)^{-\gamma}, \\
M_{3 d}(r) & =\frac{4 \pi}{3-\gamma} \rho_{0} r_{0}^{3}\left(r / r_{0}\right)^{3-\gamma},
\end{aligned}
$$

with $1<\gamma<3$. Once projected along the line of sight, we get the projected surface mass density:

$$
\begin{aligned}
\Sigma(R) & =\Sigma_{0}\left(R / r_{0}\right)^{1-\gamma}=\rho_{0} r_{0} \sqrt{\pi} \frac{\Gamma[(\gamma-1) / 2]}{\Gamma[\gamma / 2]}\left(R / r_{0}\right)^{1-\gamma}, \\
M_{2 d}(R) & =\frac{2 \pi}{3-\gamma} \Sigma_{0} r_{0}^{2}\left(R / r_{0}\right)^{3-\gamma} .
\end{aligned}
$$

We now define a possible constant surface mass density $\Sigma_{\text {sheet }}$, and three mass measurements $M_{2 d}^{1}, M_{2 d}^{2}$ and $M_{3 d}$ at radii $R_{1}, R_{2}$ and $R_{3}$ that satisfy

$$
\begin{aligned}
& M_{2 d}^{1}=\rho_{0} A(\gamma)\left(R_{1} / r_{0}\right)^{3-\gamma}+\pi R_{1}^{2} \Sigma_{\text {sheet }} \\
& M_{2 d}^{2}=\rho_{0} A(\gamma)\left(R_{2} / r_{0}\right)^{3-\gamma}+\pi R_{2}^{2} \Sigma_{\text {sheet }} \\
& M_{3 d}=\rho_{0} B(\gamma)\left(R_{3} / r_{0}\right)^{3-\gamma} .
\end{aligned}
$$

We can readily see that only in the case of three observables can one hope to constrain all three of $\rho_{0}, \gamma$ and $\Sigma_{\text {sheet }}\left(r_{0}\right.$ being an irrelevant rescaling here). In the equations above we have substituted $A(\gamma)=r_{0}^{3} 2 \pi^{3 / 2} \Gamma[(\gamma-1) / 2] / \Gamma[\gamma / 2] /(3-\gamma)$ and $B(\gamma)=r_{0}^{3} 4 \pi /(3-\gamma)$ for brevity. Schematically we thus obtain

$$
\begin{aligned}
\frac{\Gamma[(\gamma-1) / 2]}{\Gamma[\gamma / 2]} & =\frac{2}{\sqrt{\pi}} \frac{R_{3}^{3-\gamma}}{M_{3 d}} \frac{R_{2}^{2} M_{2 d}^{1}-R_{1}^{2} M_{2 d}^{2}}{R_{2}^{2} R_{1}^{3-\gamma}-R_{1}^{2} R_{2}^{3-\gamma}}, \\
\Sigma_{\text {sheet }} & =\frac{1}{\pi} \frac{M_{2 d}^{1} R_{2}^{3-\gamma}-M_{2 d}^{2} R_{1}^{3-\gamma}}{R_{1}^{2} R_{2}^{3-\gamma}-R_{2}^{2} R_{1}^{3-\gamma}},
\end{aligned}
$$

where Eq. (A.8) allows us to solve for $\gamma$ and then Eq. (A.9) yields $\Sigma_{\text {sheet. }}$

\section{References}

Auger, M. W. 2008, MNRAS, 383, L40

Auger, M. W., Fassnacht, C. D., Abrahamse, A. L., Lubin, L. M., \& Squires, G. K. 2007, AJ, 134, 668

Bolton, A. S., Burles, S., Koopmans, L. V. E., Treu, T., \& Moustakas, L. A. 2006, ApJ, 638, 703

Bolton, A. S., Burles, S., Koopmans, L. V. E., et al. 2008a, ApJ, 682, 964

Bolton, A. S., Treu, T., Koopmans, L. V. E., et al. 2008b, ApJ, 684, 248

Bolzonella, M., Miralles, J.-M., \& Pelló, R. 2000, A\&A, 363, 476

Cabanac, R. A., Alard, C., Dantel-Fort, M., et al. 2007, ApJ, 461, 813

Chabrier, G. 2005, in The Initial Mass Function 50 Years Later, ed. E. Corbelli, F. Palla, \& H. Zinnecker, Astrophysics and Space Science Library, 327, 41

Czoske, O., Barnabè, M., Koopmans, L. V. E., Treu, T., \& Bolton, A. S. 2008, MNRAS, 384, 987

Dobke, B. M., King, L. J., \& Fellhauer, M. 2007, MNRAS, 377, 1503
Fassnacht, C. D., \& Lubin, L. M. 2002, AJ, 123, 627

Fassnacht, C. D., McKean, J. P., Koopmans, L. V. E., et al. 2006, ApJ, 651, 667 Faure, C., Kneib, J.-P., Covone, G., et al. 2008a, ApJS, 176, 19

Faure, C., Kneib, J.-P., Hilbert, S., et al. 2008b, [arXiv: 0810.4838]

Gavazzi, R., Treu, T., Koopmans, L. V. E., et al. 2008, ApJ, 677, 1046

Gavazzi, R., Treu, T., Rhodes, J. D., et al. 2007, ApJ, 667, 176

Geach, J. E., Simpson, C., Rawlings, S., Read, A. M., \& Watson, M. 2007, MNRAS, 873

Geiger, B. \& Schneider, P. 1998, MNRAS, 295, 497

Gerhard, O., Kronawitter, A., Saglia, R. P., \& Bender, R. 2001, AJ, 121, 1936 Inada, N., Oguri, M., Becker, R. H., et al. 2008, AJ, 135, 496

Jullo, E., Kneib, J.-P., Limousin, M., et al. 2007, New J. Phys., 9, 447

Kassiola, A., \& Kovner, I. 1993, ApJ, 417, 450

Keeton, C. R., Kochanek, C. S., \& Seljak, U. 1997, ApJ, 482, 604

King, L. J. 2007, MNRAS, 382, 308

Kochanek, C. S. 1994, ApJ, 436, 56

Kochanek, C. S. 2002, ApJ, 578, 25

Kochanek, C. S., \& Blandford, R. D. 1991, ApJ, 375, 492

Kochanek, C. S., Falco, E. E., Impey, C. D., et al. 1999, in After the Dark Ages: When Galaxies were Young (the Universe at $2<Z<5$ ), ed. S. Holt, \& E. Smith, AIP Conf. Ser., 470, 163

Kochanek, C. S., \& Schechter, P. L. 2004, in Measuring and Modeling the Universe, ed. W. L. Freedman, 117

Koopmans, L. V. E. 2006, in Engineering and Science, ed. G. A. Mamon, F. Combes, C. Deffayet, \& B. Fort, EAS Publ. Ser., 20, 161

Koopmans, L. V. E., Treu, T., Bolton, A. S., Burles, S., \& Moustakas, L. A. 2006, ApJ, 649, 599

Limousin, M., Kneib, J.-P., \& Natarajan, P. 2005, MNRAS, 356, 309

Limousin, M., Kneib, J. P., Bardeau, S., et al. 2007a, A\&A, 461, 881

Limousin, M., Sommer-Larsen, J., Natarajan, P., \& Milvang-Jensen, B. 2007b, [arXiv: 0706.3149]

Limousin, M., Cabanac, R., Gavazzi, R., et al. 2008, [arXiv: 0812 . 1033]

Marshall, P. J., Hogg, D. W., Moustakas, L. A., et al. 2008, ArXiv e-prints

Miralda-Escudé, J. 1995, ApJ, 438, 514

Momcheva, I., Williams, K., Keeton, C., \& Zabludoff, A. 2006, ApJ, 641, 169

Myers, S. T., Jackson, N. J., Browne, I. W. A., et al. 2003, MNRAS, 341, 1

Narasimha, D., Subramanian, K., \& Chitre, S. M. 1984, MNRAS, 210, 79

Natarajan, P., \& Kneib, J.-P. 1997, MNRAS, 287, 833

Natarajan, P., Kneib, J.-P., \& Smail, I. 2002a, ApJ, 580, L11

Natarajan, P., Kneib, J.-P., \& Smail, I. 2002b, ApJ, 580, L11

Natarajan, P., Loeb, A., Kneib, J.-P., \& Smail, I. 2002c, ApJ, 580, L17

Natarajan, P., Kneib, J.-P., Smail, I., et al. 2007, [arXiv: 0711.4587]

Navarro, J. F., Frenk, C. S., \& White, S. D. M. 1997a, ApJ, 490, 493

Navarro, J. F., Frenk, C. S., \& White, S. D. M. 1997b, ApJ, 490, 493

Newton, E. R., Marshall, P. J., \& Treu, T. 2008, ArXiv e-prints

Oguri, M. 2006, MNRAS, 367, 1241

Peng, C. Y., Ho, L. C., Impey, C. D., \& Rix, H.-W. 2002, AJ, 124, 266

Sand, D. J., Treu, T., Smith, G. P., \& Ellis, R. S. 2004, ApJ, 604, 88

Schlegel, D. J., Finkbeiner, D. P., \& Davis, M. 1998, ApJ, 500, 525

Simpson, C., Martínez-Sansigre, A., Rawlings, S., et al. 2006, MNRAS, 372, 741

Steidel, C. C., Hunt, M. P., Shapley, A. E., et al. 2002, ApJ, 576, 653

Treu, T., Ellis, R. S., Kneib, J.-P., et al. 2003, ApJ, 591, 53

Treu, T., Gavazzi, R., Gorecki, A., et al. 2008, ApJ, accepted, [arXiv: 0806.1056]

Treu, T., Koopmans, L. V., Bolton, A. S., Burles, S., \& Moustakas, L. A. 2006, ApJ, 640, 662

Treu, T., \& Koopmans, L. V. E. 2002, MNRAS, 337, L6

Treu, T., \& Koopmans, L. V. E. 2004, ApJ, 611, 739

Treu, T., Stiavelli, M., Bertin, G., Casertano, S., \& Møller, P. 2001, MNRAS, 326, 237

Trujillo, I., Burkert, A., \& Bell, E. F. 2004, ApJ, 600, L39

Tu, H., Limousin, M., Fort, B., et al. 2008, MNRAS, 476

Williams, K. A., Momcheva, I., Keeton, C. R., Zabludoff, A. I., \& Lehár, J. 2008, ApJ, 672, 733

Yoo, J., Kochanek, C. S., Falco, E. E., \& McLeod, B. A. 2006, ApJ, 642, 22 